\title{
REGARDS CRITIQUES EN SUPPLY CHAIN MANAGEMENT
}

\author{
Christelle CAMMAN ${ }^{1}$ et Anne TOUBOULIC ${ }^{2}$
}

\author{
${ }^{1}$ Centre de REcherche sur le Transport et la LOGistique - Aix Marseille Univ \\ christelle.ledi@univ-amu.fr \\ ${ }^{2}$ Nottingham University Business School - Division of Operations Management and \\ Information Systems \\ anne.touboulic@nottingham.ac.uk
}

\section{Résumé}

Cet article se propose d'approfondir la réflexion en Supply Chain Management (SCM) sur les contributions potentielles des Critical Management Studies (CMS). Celles-ci invitent à une réflexivité radicale interrogeant les fondements ontologiques et épistémologiques des recherches en SCM et la prise en compte des dimensions sociales et humaines. Face à une soussocialisation des conceptualisations jusqu'à présent produites, les CMS, dans leur diversité, offrent des pistes stimulantes pour les enrichir et appréhender surtout la dynamique des SC et SCM.

\begin{abstract}
The purpose of this article is to deepen the conceptual connections between the fields of Supply Chain Management (SCM) and Critical Management Studies (CMS). We interrogate the current state of SCM through a critical lens, which entails adopting a radical reflexive approach to questioning the underlying ontological and epistemological assumptions in SCM research. In particular, we are interested in the ways in which social and human dimensions have been considered in previous research. In shedding light on the under-socialised conceptualisations of SC and SCM, we show that CMS, in their diversity, provide fruitful avenues to enhance our understanding of SC and SCM phenomena.
\end{abstract}

\section{Mots clés}

Supply Chain Management, Critical Management Studies 


\section{Introduction}

Les Critical Management Studies (CMS) interrogent les structures de domination perpétuées par le management au sein des organisations. Dans une démarche réflexive radicale (Alvesson et Sköldberg, 1999), elles évaluent les pratiques managériales au regard de critères éthiques comme la justice, l'équité ou le développement humain, en considérant les individus et groupes souvent ignorés par la recherche en management. En dénaturalisant les organisations et en dévoilant les mécanismes génératifs de structures considérées comme « oppressives », elles ont pour projet d'identifier les possibles voies de changement.

La recherche en Supply Chain Management (SCM), comme d'autres disciplines des sciences de gestion, a privilégié un intérêt de connaissance instrumental (Habermas, 1979) visant, par le déploiement d'outils de gestion, à maîtriser les flux physiques tout au long de la supply chain (SC) pour en améliorer la performance globale (coût, niveau de service, délai, flexibilité, réactivité). Cette maîtrise, du fait de la fragmentation des chaînes de valeur, suppose de coordonner un nombre croissant de parties prenantes. Agile, lean ou leagile, le management des SC s'adapte aux caractéristiques environnementales (variabilité de la demande, chronocompétition) en s'appuyant, dans des démarches plus ou moins collaboratives, sur un ensemble de dispositifs juridiques, techniques et organisationnels qui ont conduit les chercheurs en SCM, issus de ses diverses disciplines originelles (marketing, logistique, gestion des opérations, achats), à emprunter nombre de concepts et théories à d'autres disciplines (stratégie, organisation, systèmes d'information, contrôle de gestion, gestion des ressources humaines, organisation, stratégie). $\mathrm{Si}$, dans ces disciplines originelles et d'emprunt, un courant critique s'est développé depuis les années 1990 (Golsorkhi et al, 2009), interrogeant notamment les conséquences de ces dispositifs sur les individus et les groupes, les organisations et/ou la société, il n'en est pas de même en SCM.

Cet article souhaite donc approfondir la réflexion en SCM sur les contributions potentielles des CMS. Leur questionnement sur la participation de la science à la production des systèmes sociaux invite, dans la première partie, à s'intéresser aux conceptualisations des SC et du SCM produites jusqu'à présent en focalisant sur la façon dont y ont été intégrées les dimensions sociales. Si les SC sont généralement conceptualisées comme des systèmes complexes et le SCM comme l'ensemble des pratiques déployées pour les piloter, la compréhension de leur émergence, diffusion et évolution reste sous-socialisée. La seconde partie se propose alors, après avoir présenté les principaux paradigmes et perspectives théoriques qui structurent le champ des CMS, de discuter de leurs apports potentiels pour enrichir les conceptualisations des $\mathrm{SC}$ et du SCM et la compréhension de leur production sociale invitant aussi à une réflexion sur la performativité critique des recherches et l'engagement des chercheurs (Spicer et al, 2009).

\section{I - UNE SOUS-SOCIALISATION DES RECHERCHES EN SCM}

Majoritairement fonctionnalistes, les conceptions des SC et les dispositifs de gestion promus pour les piloter (1.1) ont conduit à une prise en compte limitée, purement stratégique, des dimensions sociales. Ces perspectives ont ainsi peu ou prou éludé la dynamique des SC comprises comme des systèmes sociaux (1.2). La prise en compte du pouvoir dans les SC, thématique importante en SCM et fondatrice des CMS, permet d'illustrer cette limite au regard de travaux plus récents, ancrés en théorie des organisations qui, sans s'inscrire dans des perspectives critiques mais en leur ouvrant la voie, soulignent l'intérêt d'enrichir les conceptions des $\mathrm{SC}$ et du SCM, de même que les approches théoriques et méthodologiques habituellement mobilisées (1.3).

\section{1 - La Supply Chain, un système complexe}


Le SCM coordonne, par divers dispositifs de gestion plus ou moins collaboratifs, les processus transversaux contribuant à la création de valeur globale de la SC (gestion de la relation client, du service client, de la demande, des commandes, de la relation fournisseur, des retours, planification, développement de nouveaux produits / services, commercialisation, finance). Ces processus sont conjointement pilotés par les équipes fonctionnelles (marketing / vente, logistique, production, achats) des différentes organisations (fournisseurs, sous-traitants, industriels, prestataires logistiques, distributeurs) participant à la production et à la fourniture d'une offre produits / services associés répondant aux attentes d'un client.

$\mathrm{Au}$ regard de ses enjeux stratégiques et de son périmètre, diverses disciplines, éponymes des fonctions précitées, se sont appropriées, tout en restant cloisonnées, les problématiques liées au déploiement du SCM et ont participé à la structuration d'un champ de recherche partageant la même représentation des SC (Livolsi, 2009). Celle-ci, fondée sur les flux physiques, informationnels et financiers permet d'en identifier les composantes (entreprises, processus, fonctions, individus), leurs interactions ou interfaces, ainsi que celles avec son environnement. Les processus de décision multidimensionnels et multi-acteurs ont conduit les chercheurs à mettre l'accent sur les échanges et le traitement des informations et, a fortiori, sur la dimension technologique des SC en s'appuyant notamment sur une approche systémique originellement inspirée de la cybernétique. Si, au début des années 2000, un changement paradigmatique s'esquisse avec l'émergence mais lente diffusion, dans les pays anglo-saxons, des «systèmes adaptatifs complexes » (Choi et al, 2001) et en France, de la "pensée complexe » (Bruyère et Fabbe-Costes, 2011), la discipline reste encore très fortement imprégnée de cette conception fonctionnaliste des SC (Burgess et al, 2006).

Celle-ci privilégie l'unité fonctionnelle du système que représente la $\mathrm{SC}$ alors maintenue en équilibre par le biais d'ajustements relatifs à des dysfonctions. Les dimensions physiques, techniques, sociales et humaines y sont paradoxalement le plus souvent traitées séparément et « manipulées » pour atteindre les objectifs globaux de performance. L'intérêt de connaissance instrumental dominant réifie les rapports sociaux dans des travaux qui ont produit, quel que soit le processus concerné, nombre de prescriptions sur les modalités formelles ou informelles de coordination et les dispositifs de gestion à promouvoir, l'orientation des relations ou les modes de gouvernance des SC à privilégier.

Les « systèmes complexes adaptatifs », et a fortiori la « pensée complexe », au regard de la prise en compte des phénomènes d'émergence et de l'impossibilité de prévoir la réaction du système, s'intéressent alors aux caractéristiques de ces dispositifs pour maintenir sa contrôlabilité en préservant un certain degré d'autonomie de ses composantes, gage d'adaptation vis-à-vis de l'environnement. En réhabilitant le rôle des acteurs (individuels - dont les chercheurs - et collectifs) dans la production des SC, par le biais des représentations qu'ils en conçoivent, fonction aussi de leurs projets et valeurs, ces perspectives se sont focalisées sur les règles et principes permettant justement de se représenter leur complexité en mettant alors l'accent sur les dimensions cognitives et/ou communicationnelles. Les prescriptions managériales concernent ainsi, dans une perspective plus pragmatique, le déploiement de dispositifs (systèmes d'information et de communication notamment) et démarches organisationnelles (système de veille, empowerment, etc.) facilitant l'intercompréhension, la construction collective de sens et la prise de décision. La régulation y est toujours privilégiée, valorisant l'ordre, le consensus, la solidarité, l'intégration (Burell et Morgan, 1979), mais les dimensions sociales y restent peu explorées appelant d'autres conceptualisations des SC et du SCM, et d'autres théories que celles mobilisées dans la majorité des recherches (coût de transaction, approche ressources- compétences) pour enrichir leur compréhension.

\section{2 - Sur l'intégration des dimensions sociales en SCM}

Les dimensions physique et technologique occupent donc un pan important de la recherche en SCM qui s'est toujours rapidement appropriée toute innovation permettant d'améliorer ses 
modélisations et/ou d'optimiser les processus. L'agitation actuelle sur les potentialités du big data, de l'intelligence artificielle ou des blockchains illustre cette appétence. Ce faisant, même si les conséquences de cette «technologisation» interpellent, les dimensions sociales et humaines des SC font toujours l'objet d'une moindre attention. Comme évoqué précédemment, les clôtures inhérentes aux perspectives développées dans la discipline ont laissé peu de place à une réflexion plus approfondie sur la dynamique sociale.

Les recherches sur le pouvoir, au cœur des CMS et thématique importante des travaux sur les relations inter-organisationnelles au sein des SC, illustrent ces perspectives et la soussocialisation qui en résulte. Dans leur synthèse sur le pouvoir, Reimann et Ketchen (2017) identifient les problématiques structurant la compréhension de cette dimension des SC. Après avoir défini le pouvoir (formes, modalités d'exercice, sources), les chercheurs se sont attachés à identifier quel pouvoir (coercitif, non coercitif, d'expertise, de légitimité, etc.) avait quel(s) impact(s) sur les relations au sein de dyades voire de triades et in fine, sur la performance afin d'orienter notamment les modalités de gestion de la relation fournisseurs.

Les travaux sur le pouvoir, comme ceux sur la confiance, autre dimension importante des SC, ont principalement mobilisé des perspectives théoriques fondées sur les ressources comme la théorie relationnelle de Dyer et Singh (1998). Dans ce cadre, le périmètre de la SC est réduit (dyade) et la prise en compte des dimensions relationnelles informelles s'inscrit toujours dans une logique "fin-moyen" contribuant à l'effort stratégique d'amélioration de la performance opérationnelle et, in fine, financière. La quête d'une rente relationnelle constitue la principale motivation des entreprises. La confiance, concept dont la définition est souvent absente ou construite de façon réductible à partir de l'agrégation d'aspects quantifiables, ou encore le capital social (mesuré au regard des liens tissés par les entreprises, de leur intensité et de leur activation plus ou moins rapide pour pallier un dysfonctionnement ou dans le cadre d'une reconfiguration de la SC) sont conçus, à l'instar du pouvoir, comme des processus d'accumulation de ressources.

Gérées stratégiquement par les entreprises, ces ressources dévoilent cependant les jeux de pouvoir, en particulier des acteurs en position dominante, visant à influencer les processus décisionnels et organisationnels des autres acteurs de la SC. Cox (2001) et Cox et Chicksand (2007), en s'appuyant sur la théorie de la dépendance des ressources, montrent qu'en détenant des ressources critiques dont les autres dépendent, les acteurs dominants maintiennent volontairement des déséquilibres pour préserver leur position au sein de la SC. Ainsi, même des démarches collaboratives, comme par exemple le category management fondé sur un partage d'informations stratégiques entre industriels et distributeurs (études de marché), peuvent être comprises comme un moyen pour ces derniers de capter des ressources critiques afin d'accroître leur pouvoir de marché et in fine, de négociation (Camman et Livolsi, 2009). L'engagement dans des démarches collaboratives repose donc sur une analyse fine de l'interdépendance entre acteurs, des structures de pouvoir sous-jacentes et de leur évolution.

L'absence de perspectives diachroniques occulte cependant cette dynamique. Quelques récents travaux en SCM sur le dark side des relations inter-organisationnelles commencent toutefois à s'y intéresser. La «corrosion des relations » au sein d'une dyade et de la performance visée (Villena et al, 2015) y est comprise comme le résultat de divers comportements (réduction des conflits, confiance excessive et manque d'objectivité, opportunisme, redondance de l'information). La gestion des relations doit donc intégrer et atténuer ce risque de corrosion en déployant des dispositifs contractuels et des démarches appropriées comme la veille relationnelle, l'animation conjointe de la performance ou encore, une rotation du personnel, en particulier des boundary-spanning managers, pour limiter la « camaraderie ». Ces précautions prises, cette sombre dimension des relations peut alors être partiellement tolérée ! (op.cit, 2015).

Le dark side ne s'appréhende pas cependant qu'à l'examen des comportements au sein d'une dyade. D'autres facteurs organisationnels et environnementaux participent de cette corrosion 
des relations (Oliveira et Lumineau, 2019). Ils appellent non seulement des perspectives multiniveaux (individus, organisations, dyades, triades, SC, environnement) mais une plus grande attention aussi aux multiples sphères (politique, juridique, sociale, culturelle, etc.) dans lesquelles ces relations sont elles-mêmes encastrées. Ces travaux sur le dark side en SCM, peu développés encore et fonctionnalistes, peuvent cependant ouvrir la voie à une compréhension renouvelée et plus socialisée des $\mathrm{SC}$ et de leur dynamique.

\section{3 - Sur la production / reproduction des SC}

Reconnaissant la nature émergente du SCM et par-là, les dimensions contextuelles mais aussi les interactions entre acteurs qui participent de sa production, la conception des SC comme "systèmes adaptatifs complexes » (Choi et al, 2001), en élargissant les périmètres et unités d'analyse et en adoptant des perspectives multi-niveaux, permet d'appréhender les dynamiques sous-jacentes au succès ou à l'échec de nouvelles pratiques dans un contexte interorganisationnel étendu comme par exemple, celles visant à réduire l'empreinte carbone au sein des SC alimentaires (Touboulic et al, 2018).

Cette conceptualisation et analyse des SC permet de dépasser les critiques liées au foisonnement de travaux sur les dyades qui tendent à en faire le niveau explicatif des SC, appauvrissant, comme le souligne Lecocq (2002), la compréhension de leur dynamique en l'absence de prise en compte des éléments de contexte (biais atomistique) ou des interactions situées à d'autres niveaux (biais écologique). Comme évoqué précédemment, la compréhension de la dynamique fondée sur les principes de complexité (auto-organisation, récursivité, dialogique, écologie de l'action, hologrammique, etc.) mérite toutefois d'être enrichie (1.1). Les théories des organisations, notamment celles d'inspiration sociologiques ayant longuement débattu sur la conception et l'évolution des systèmes sociaux, peuvent dès lors contribuer à cet enrichissement en ouvrant aussi la voie à une réflexivité plus radicale.

En mobilisant des perspectives néo-institutionnelles sociologiques, certains interrogent la diffusion de pratiques collaboratives au sein des SC. Fernie et Tennant (2013) soulignent ainsi l'influence des accords-cadres sur l'intégration clients-fournisseurs dans le secteur de la construction et l'émergence de clusters au sein desquels un mode de gouvernance clanique est privilégié. Pour autant, ils montrent que les valeurs prévalant dans le secteur (économie de marché, rapport coût - avantage) et l'absence de "champion » (les supply chain managers n'existent pas dans le secteur) limitent l'adoption de pratiques en SCM plus collaboratives. Portant sur la « contagion du SCM», le travail de McFarland et Payan (2008), réalisé à partir de l'analyse de triades dans divers secteurs d'activité, témoigne de l'influence de l'orientation des relations dyadiques et des boundary spanning managers dans la diffusion de pratiques en SCM. Ces derniers jouent effectivement un rôle de traducteur des objectifs de leur organisation, en diffusent les normes en s'assurant aussi de leur respect. Les dimensions psychologiques les caractérisant (cognition, motivation, valeurs, etc.) influencent le développement des relations et la diffusion des pratiques au niveau local. Une analyse plus fine des interactions aux niveaux individuel et collectif (équipe SC ou entreprise) et entre ces niveaux, intégrant les dimensions psychologiques, mais aussi les variables environnementales et organisationnelles, est donc préconisée dans les travaux sur le behavioral SCM (Schorsch et al, 2017).

Ces recherches invitent à dépasser le cadre restreint de la dyade pour mieux saisir les processus d'adoption et diffusion de pratiques en SCM en interrogeant les interactions entre représentations des acteurs à différents niveaux, valeurs et normes (organisationnelles et sectorielles) orientant les comportements et structures de pouvoir.

En adoptant aussi une « approche méso », Camman et al (2013) confirment l'influence, sur la diffusion de la Gestion Mutualisée des Approvisionnements (GMA) dans le secteur de la distribution, des jeux de pouvoir et surtout des discours qu'en ont produits les diverses parties 
prenantes (industriels, distributeurs, prestataires logistiques, consultants, think tank, Etat). Ceux-ci, en mettant en exergue soit sa complexité conceptuelle et opérationnelle, soit ses gains économiques potentiels, soit ses enjeux environnementaux, n'ont pas produit une théorisation suffisante pour qu'elle se diffuse alors que sa pertinence économique et environnementale était avérée. Ce travail souligne, qu'au-delà des acteurs traditionnels de la SC (industriels, distributeurs, prestataires), d'autres peuvent influencer la diffusion de nouvelles démarches en SCM et participent donc de leur production. L'examen des pratiques individuelles ou collectives, élargi à l'échelle d'un secteur d'activité ou d'une filière, peut ainsi s'avérer fécond pour en appréhender les processus de signification et de légitimation.

Cette compréhension suppose toutefois des approches plus historiques, diachroniques et démystificatrices. Les CMS, en s'attachant justement à dévoiler ces opérations de mystifications, offrent des perspectives intéressantes, en particulier face aux injonctions de plus en plus paradoxales auxquelles chercheurs comme professionnels sont confrontés.

\section{2 - VERS UN SCM CRITIQUE}

Qu'il s'agisse du déploiement de nouvelles technologies, de responsabilité sociétale de l'entreprise ou de développement durable, les entreprises sont aujourd'hui questionnées sur leur rôle dans la société en particulier au regard de leur participation à la production des normes et des valeur(s). Les chercheurs en SCM aussi! Les CMS, quel que soit leur ancrage paradigmatique (2.1), peuvent, en explorant ces logiques de création / destruction de normes et de valeur(s), s'avérer fécondes pour dépasser les clôtures des perspectives majoritairement mobilisées en SCM en contribuant à leur enrichissement conceptuel, théorique et méthodologique (2.2) et pour penser le changement tant dans les pratiques professionnelles que scientifiques en engageant aussi une réflexion sur le rôle des chercheurs (2.3).

\section{1 - Sur les ancrages paradigmatiques des CMS}

Les concepts (SC, SC Orientation, SC Integration, etc.), classifications (grand acheteur, donneur d'ordres, acteur pivot, etc.), prétentions à la validité (création globale de valeur, solidarités de chaîne...), de même que les modalités de coordination tant formelles qu'informelles et les dispositifs de gestion ne sont neutres ni dans leur genèse, ni dans leurs effets dans la production des systèmes sociaux. La réflexivité consiste alors à questionner les présupposés sur lesquels se fondent la connaissance et les pratiques en SCM et les conditions de leur détermination dans des contextes historiques et sociaux spécifiques. Cette dénaturalisation des processus managériaux repose sur le dévoilement des dynamiques de création, de maintien ou de destruction de pratiques institutionnalisées dans une perspective non fonctionnaliste. Les théories et méthodologies mobilisées, ainsi que les possibilités offertes en termes de changement, de cette opération de dénaturalisation, témoignent cependant d'ancrages paradigmatiques différents au sein même des CMS. Adler et al (2007) en identifient trois principaux dont les fondamentaux sont ici résumés : standpoint theory, post-modernisme critique et réalisme critique.

Dans le premier, standpoint theory, porté par le courant féministe et d'inspiration marxiste, les chercheurs déconstruisent les faits sociaux à partir du point de vue d'une catégorie d'individus, ceux qui sont opprimés, exploités. Le projet politique des chercheurs y est assumé avec, dans une perspective plus ou moins activiste, la volonté de faire valoir ces points de vue (celui des femmes, des diverses ethnies, etc.), considérés, vu leur place dans la société, comme plus à même d'en divulguer les mécanismes de production et de faire valoir d'autres trajectoires soustraites par les discours (des) dominants. Les logiques de domination, luttes de pouvoir sont 
donc prégnantes dans ces analyses qui dévoilent leur processus de production par les institutions, y compris la science.

Si elles sont intéressantes pour appréhender certains mécanismes génératifs des systèmes sociaux, ces perspectives ne sont pas exemptes de critiques. La sur-socialisation, fondant l'identité sur l'appartenance à un groupe social (classe, genre, culture ou race) au sein duquel les membres partageraient les mêmes intérêts, fait courir le risque d'une généralisation et d'un déterminisme excessif. En s'éloignant du vécu, la distanciation et le surplomb peuvent s'avérer peu pragmatiques, générer plus de frustration voire exacerber les conflits dans une radicalisation possible des points de vue liée au rejet de tout relativisme, contrairement au postmodernisme critique.

Ce second paradigme critique considère les organisations comme le produit temporaire des discours fragmentés des acteurs quelle que soit leur position dans le système social. Dans une perspective derridienne, les organisations, les individus, de même que leurs relations, effets du langage, sont donc indéterminés. L'enjeu consiste ici à "ouvrir les indéterminations que la science sociale moderne, les conceptions quotidiennes, les routines, et les pratiques ont fermées » (Alvesson et Deetz, 1996, p. 210).

Ce relativisme, qui rend aussi le consensus impossible, implique pour le chercheur une réflexivité permanente, y compris dans la diffusion de ses analyses (forme et contenu) "pour inviter le lecteur au dialogue et à la réflexion » en évitant de clôturer a priori le sens (AllardPoési et Perret, 2002, p 22). Il interpelle toutefois, notamment en sciences de gestion, sur les possibilités d'action offertes quand tout doit être en permanence mis en doute et que le couplage entre connaissance et pratique est alors fondamentalement aléatoire (Allard-Poési et al, 2015).

Le troisième paradigme, réaliste critique, issu de la philosophie de Roy Bashkar (1979) et repris par Archer (1995), offre une alternative à ce relativisme. Ce sont ici les mécanismes génératifs, les structures, les pratiques et les rôles qui sont au cœur des analyses. Dans cette perspective, inspirée aussi des travaux de Bourdieu, Foucault sur la relation savoir-pouvoir, Gramsci sur l'hégémonie ou Giddens avec sa théorie de la structuration, le réel (structure sociale) existe indépendamment du sujet observant, connaissant et agissant. Ces structures sont cependant transformées dans l'interaction par les individus. Elles sont donc le médium et le résultat de l'action (Giddens, 1984). Dans une perspective critique, le caractère contraignant des structures est mis en exergue. L'émancipation des acteurs, vis-à-vis de ces contraintes structurelles, repose sur une démarche réflexive (compréhension de l'élaboration structurale) que les chercheurs encouragent en restant distanciés ou en intervenant directement dans le système social.

Ces trois paradigmes critiques peuvent enrichir la recherche en SCM. Une relecture critique des travaux réalisés sur certains concepts est déjà une piste pour dévoiler leurs présupposés, mais aussi leur contribution à la production des démarches de SCM face, notamment, aux problématiques rencontrées dans leur déploiement par les professionnels et qui témoignent de l'incomplétude des perspectives instrumentales. La conduite de recherches critiques est une autre voie à peine empruntée par la discipline. Le paragraphe suivant s'y consacre en identifiant les apports des rares travaux existants ainsi que, dans leur prolongement, les pistes de recherche potentielles.

\section{2 - Perspectives critiques en SCM}

Le premier paradigme (standpoint theory) renvoie à une critique du SCM généralement conduite dans d'autres disciplines (géographie économique et sociale, philosophie ou sociologie). Ces travaux interrogent les idéologies sous-jacentes à la conception et au pilotage des SC et mettent en lumière leur participation à la production de systèmes socio-économiques inégalitaires. Ils s'intéressent ainsi particulièrement aux effets de l'éclatement des processus 
productifs, de leur maîtrise et compression spatio-temporelle accrues (Harvey, 1989) sur les relations d'emploi, la précarisation de toute une partie de la population active, mais aussi la construction des identités individuelles et collectives, la mise à mal des solidarités, etc. (Nielson, 2014 ; Tsing, 2009).

Ces questionnements, même à l'échelle d'entreprises ou de dyades, ont peu mobilisé les chercheurs en SCM, y compris en $S C$ durable. Tout en restant déterministes, ces perspectives peuvent enrichir certains concepts en SCM comme le behavioral SCM (1.3) ou le Dark Side (1.2) en interrogeant les conséquences de pratiques interentreprises négatives sur les comportements des acteurs, le développement de contre-pouvoirs (Noireaux et Poirel, 2014).

Les logiques de captation de valeur maintenant certaines catégories d'acteurs (prestataires logistiques, transporteurs, petits sous-traitants ou fournisseurs) dans des situations précaires (du point de vue du maintien de leurs marges) incitent à approfondir les recherches sur la création de valeur(s) au sein des SC en y intégrant d'autres valeurs que financière. Elles invitent déjà à considérer tous les acteurs d'une $\mathrm{SC}$ et à questionner les catégorisations qui ont aussi conduit à réduire les périmètres d'analyse en focalisant l'attention, selon les disciplines, sur quelques dyades quasiment idéalisées (grands acheteurs et fournisseurs stratégiques, firme pivot et équipementiers, donneurs d'ordres et prestataires) véhiculant une vision des SC et du SCM en décalage avec le vécu de ceux qui sont peu ou pas intégrés dans le pilotage des flux.

New (2004) mettait déjà en garde les chercheurs, via leur rhétorique de la chaîne (solidarité, co-création de valeur, performance globale, etc.), sur leur participation à la légitimation de pratiques en contradiction avec les valeurs promues. Rhétorique des chercheurs et des professionnels qu'Higgins et Ellis (2009) proposent d'analyser pour mettre à jour les problématiques éthiques et morales au sein des SC. Gold et Schleper (2017), en s'appuyant sur le concept de réification d'Honneth, interpellent aussi sur ces classifications et les clôtures qu'elles produisent et préconisent un changement paradigmatique par la prise en compte des représentations de toutes les parties prenantes dans les décisions stratégiques et opérationnelles au sein des SC, en particulier lorsqu'il s'agit de construire des SC durables.

En considérant que les structures de domination au sein des SC clôturent la connaissance produite et qu'il est nécessaire de les mettre à jour avec leurs conséquences humaines et sociales, ces derniers travaux, de nature interprétativiste ou constructiviste, appellent donc un changement de paradigme. Le post-modernisme critique auquel ils se rattachent, en prenant en compte l'altérité de l'autre, ses identités multiples quelle que soit sa position dans le système social, relativise les grands récits souvent unilatéraux du standpoint ou des approches marxistes, dénonce les catégorisations et la croyance dans le progrès en considérant la réalité sociale comme le produit temporaire et indéterminé des discours et représentations des acteurs. En adoptant des approches discursives ancrées dans les théories féministes de la nouvelle génération ou postcoloniales, certains auteurs s'intéressent ainsi aux conséquences de pratiques à visée optimisatrice en SCM, comme les démarches de RSE déployées dans les SC globales. Ces recherches soulignent, entre autres, que le contrôle des pratiques sociales des fournisseurs (audit, code de conduite, etc.), sous-tendu par des logiques d'exploitation, maintient les disparités de pouvoir au sein des SC et participe aussi de la marginalisation des travailleurs par une conception réductrice de leurs rôles (Barrientos et al, 2003 ; Prieto-Carron 2008). Elles interrogent aussi, au regard du caractère performatif du discours, la construction du sens. Ainsi, McCarthy et al (2018) conduisent, au sein d'une SC d'une firme multinationale agroalimentaire, une analyse critique de l'influence de son discours sur le développement des petits agriculteurs. Ils mettent en lumière les contradictions entre le discours unilatéral de la multinationale et ses pratiques comme l'empowerment. Fondé sur l'émancipation et la capacité à gérer son propre destin, celui-ci est positivement valorisé par le groupe industriel en occultant toutefois la situation réelle et les aspirations des agriculteurs, et soutenu, dans une perspective instrumentale, en phase avec ses propres objectifs de performance. 
En SC durable, la prise en compte de voix multiples, au-delà de celles dominantes des grandes entreprises ou firmes-pivots, permet donc d'explorer les paradoxes et les contradictions, les logiques sous-jacentes à la réalisation des arbitrages et des compromis. Elle vise un dépassement des approches qui privilégient toujours la recherche de stratégies «gagnantgagnant » fondées sur un principe d'efficience avec une considération finalement marginale des enjeux écologiques ou sociaux.

Méthodologiquement, ces analyses des discours, des textes peuvent rester distanciées. Il peut s'agir de la lecture critique de travaux en SCM mobilisant des perspectives théoriques telles que celles déjà évoquées ou encore les genders studies ou cultural studies pour décrypter les normes et valeurs qui prévalent, participent de la signification et de la légitimation des pratiques en orientant, par exemple, la gestion des relations entre parties prenantes. Pour faire évoluer ces pratiques, la conduite de recherches-action critiques est aussi préconisée (Touboulic et Walker, 2015) en participant, dans des démarches réflexives, à la résolution de problèmes perçus par les acteurs. L'enjeu consiste alors à identifier les interprétations et représentations multiples, leurs fondements, mais aussi les jeux de pouvoir, valeurs, normes, etc. qui limitent l'expression de toutes les voix et/ou clôturent a priori le sens.

Dans le réalisme critique, les SC ne sont pas que virtuelles. Elles préexistent aux individus et groupes. Ils peuvent en orienter la direction mais celles-ci, comme totalité, leur échappent. L'objectif réside dans l'explication des faits en mettant à jour, par abduction notamment, leurs mécanismes génératifs (Rotaru et al, 2014). Enrichissant la conceptualisation des SC, une ontologie stratifiée met en exergue un pouvoir causal des structures indépendant de celui des individus. Celle-ci est opportune pour saisir, au regard de l'antériorité des structures et de leur transformation progressive par les acteurs, les phénomènes de diffusion et appropriation des pratiques en SCM. Aastrup et Halldórsson (2008) en soulignent la pertinence et la fertilité dans le cadre d'études de cas sans toutefois l'appliquer. Les modalités de structuration (schèmes interprétatifs, ressources mobilisées, règles et normes) observables dans l'action, peuvent effectivement dévoiler certains mécanismes génératifs. Camman et al (op.cit, 2013) montrent ainsi que la diversité des sources de légitimation (économique, environnementale) et de signification, parfois contradictoires (collaboration, coopétition, complexité opérationnelle et organisationnelle), promues par les diverses parties prenantes pour valoriser la GMA, n'ont pas permis son développement compte tenu des structures de domination existantes (1.3). Adamides et al (2012), via un outil de simulation, montrent l'influence de la structure sociale sur la reproduction de pratiques de pilotage des flux inefficientes dans les SC de produits saisonniers.

Au regard des rôles et des pratiques des acteurs de la SC, il devient possible, dans une perspective diachronique, d'identifier les mécanismes génératifs des structures sociales, les voies qui auraient pu être prises et ne l'ont pas été du fait des contraintes structurelles, mais aussi, de nouvelles trajectoires potentielles, socialement acceptables et assorties de moyens d'action crédibles dans le contexte actuel. En proposant une démarche méthodologique, la "déconstruction créatrice », Camman et Livolsi (2016) analysent la structuration de la SC au sein de la filière vitivinicole bordelaise. Ils témoignent de l'influence des structures de domination existantes sur la capacité du secteur à s'organiser pour optimiser sa création de valeur globale, mais aussi sa contribution à l'économie locale. Ils identifient, dans une perspective généalogique, les pratiques qui ont participé de leur production (classification des vins, système des primeurs, survalorisation des grands crus, vente "sortie chai », etc.) et, au regard de la dynamique actuelle, de potentielles évolutions pour la filière (logistique mutualisée, zone franche pour le stockage et le commerce, etc.) en lien avec les autres acteurs des SC vitivinicoles et, plus largement du territoire.

Les démarches méthodologiques des travaux précédemment exposés sont donc variées. Quelles qu'elles soient, le projet d'émancipation des acteurs vis-à-vis de structures sociales 
contraignantes ou oppressives, au cœur des perspectives critiques, questionne le rôle des chercheurs.

\section{3 - Sur l'engagement des chercheurs en SCM}

Si la responsabilité sociale et politique des chercheurs occupe une place centrale dans les perspectives critiques, leur engagement et posture vis-à-vis du système social font débat. Alors que la majorité des auteurs reconnaît l'importance d'une démarche réflexive, certains prônent une position de désengagement ou d'anti-engagement pour préserver la pureté de la critique et éviter ainsi les dangers d'assimilation du chercheur dans la pratique. Cependant, avec ce positionnement, "le risque est alors que les études critiques se coupent du monde des entreprises et soient un simple forum où le jargon académique, la réflexivité et la voix de l'auteur dominent" (Corbett-Etchevers, 2011, p.133). Les risques associés aux diverses postures critiques ont été précédemment évoqués. Un trop grand déterminisme ou une sursocialisation, a fortiori si l'analyse reste surplombante, sont peu pragmatiques, en particulier lorsqu'il s'agit de faire évoluer les pratiques dans et entre les entreprises. En rejetant tout relativisme, un tel positionnement peut aussi conduire, en se coupant du vécu des acteurs, à une généralisation abusive voire une radicalisation remplaçant alors une idéologie par une autre. A l'inverse, un trop grand relativisme fait aussi courir le risque d'une absence de consensus, même temporaire, et peut conduire à une impasse.

D'autres auteurs revendiquent un engagement, au sens politique, en reconnaissance de la nécessité de transformer les pratiques en SCM face aux enjeux sociaux (Meehan et al. 2016). Ceux-là soulignent l'importance d'encourager les praticiens à une réflexivité critique, notamment dans le cadre des recherches en SC durable, afin d'identifier et de remettre en question les présupposés sur lesquels se fondent leurs pratiques (Touboulic et Walker, 2015 ; Matthews et al., 2016).

Ces débats sur la participation des chercheurs en management à la transformation des pratiques et des systèmes sociaux ont particulièrement été relancés dans le cadre des travaux sur la performativité et, au sein des CMS sur la stérilité de l'anti-performativité (Fournier et Grey, 2000) remplacée par Spicer et al (2009) par la performativité critique. Celle-ci se caractérise par une posture affirmative valorisant l'existence de représentations multiples, une éthique de l'empathie (ethics of care), une orientation pragmatique, la prise en compte des potentialités et une orientation normative. Elle "implique une intervention active et subversive dans les discours et les pratiques managériaux » (p. 538). L'enjeu est alors d'identifier de nouvelles pratiques «managériales » qui génèrent un résultat en termes d'amélioration du bien-être, de reconnaissance, de capacité à choisir et décider de son destin.

De telles entreprises, compte tenu des risques qu'elles représentent pour les acteurs engagés dans ce processus réflexif, questionnent le rôle et la responsabilité du chercheur. Une éthique de l'empathie (ethics of care) est effectivement nécessaire. Elle suppose que le chercheur, comme évoqué dans les pistes de recherche potentielles (2.2), s'engage à exposer ses valeurs et ses présupposés épistémologiques, à interroger par-là même ses motivations mais aussi l'impact de ses recherches et, surtout, à prendre en considération l'ensemble des points de vue, surtout ceux des individus et groupes qui sont «systématiquement opprimés, exploités et dominés » (Corbett-Etchevers, 2011, p.134).

En conséquence, l'engagement appelle aussi une connaissance en profondeur des systèmes sociaux (SC) et donc, des perspectives théoriques à même d'en appréhender la genèse, la production et reproduction, le travail institutionnel, etc. Il invite donc, en SCM, à interroger la dynamique des SC, la légitimation des pratiques, leur institutionnalisation au regard de critères qui dépassent la simple quête d'efficience d'une ou deux parties prenantes seulement. Cette compréhension des SC et du SCM, qui appelle aussi plus de pluridisciplinarité, répond aux 
enjeux actuels auxquels les entreprises sont confrontées en tant que partie prenante d'une société qui questionne fondamentalement leur rôle et la légitimité de leurs productions.

\section{Conclusion}

Les pistes de recherche critique en SCM, présentées ici de façon synthétique, ont pour finalité d'appréhender les rapports entre connaissances et pratiques en responsabilisant les chercheurs. Elles invitent à considérer les SC et le SCM comme de véritables produits de l'activité humaine dans laquelle les chercheurs interviennent pleinement en y injectant les résultats de leurs travaux, et par laquelle ils sont influencés à chaque étape de leur démarche. Elles dévoilent les clôtures épistémologiques, théoriques ou méthodologiques qui engendrent un certain statu quo au sein de la discipline.

Les travaux présentés sont alors autant de voies possibles d'émancipation des chercheurs. Les CMS, dont les positionnements sont variés, ouvrent d'une part des voies ontologiques et épistémologiques pour enrichir les conceptualisations des SC, et d'autre part des voies théoriques (marxisme, féminisme, gender et cultural studies, réalisme critique, structuration, etc.) et méthodologiques (approches discursives, recherche-action critique, déconstruction, etc.) pour en comprendre la dynamique et faire évoluer les pratiques avec et pour tous les acteurs concernés et/ou impactés par leur pilotage.

\section{Bibliographie}

Aastrup J. et A. Halldórsson. (2008). "Epistemological role of case studies in logistics: a critical realist perspective", International Journal of Physical Distribution \& Logistics Management, vol.38, n¹0, p.746-763.

Adamides E.D., Papachristos G., Pomonis N. (2012). "Critical realism in supply chain research: Understanding the dynamics of a seasonal goods supply chain", International Journal of Physical Distribution \& Logistics Management, vol.42, n¹0, p. 906-930.

Adler P.S., Forbes L.C., Willmott H. (2007). " Critical management studies », The academy of management annals, vol.1, ${ }^{\circ} 1$, p.119-179.

Allard-Poésie F., Germain O., Huault I., Koenig G. (2015). « Les théories des organisations sont-elles toujours bien inspirées ? Quatre regards. », Economies et sociétés, Série Economie de l'entreprise, $\mathrm{n}^{\circ} 23$, p. 111-140.

Allard-Poési F. et Perret V. (2002). «Peut-on faire comme si le post-modernisme n'existait pas? », dans Questions de méthodes en sciences de gestion, Mourgues N., Allard-Poési F., Abdelmajid A., Charreire-Petit S. et Le Goff J. (Dir), EMS, p. 255 - 291.

Alvesson M. et Deetz S. (1996). "Critical Theory and Postmodernism Approaches to Organizational Studies", in Clegg, Hardy and Nord (Eds.), Handbook of Organizational Studies, Sage, London, p. 191-217.

Alvesson M. et Sköldberg K. (1999). Reflexive methodology, Sage, London.

Archer, M. (1995) Realist Social Theory: The Morphogenetic Approach, Cambridge University Press, Cambridge.

Barrientos S., Dolan C. et Tallontire A. (2003). "A gendered value chain approach to codes of conduct in African horticulture". World Development, n'31; p. 1511-1526.

Bhaskar, R. (1979), The Possibility of Naturalism, Sussex: Harvester.

Burell G. et Morgan G. (1979). Sociological paradigms and organizational analysis, Heinemann, London.

Bruyère C. et Fabbe-Costes N. (2011). "Le pilotage des chaînes multi-acteurs temporaires", Revue Française de Gestion Industrielle, vol. 30, n³, p. 61-77. 
Burgess K., P.J. Singh et Koroglu R. (2006). "Supply chain management: a structured literature review and implications for future research", International Journal of Operations and Production Management, vol. 26, nº , p. 703-729.

Camman C. et Livolsi L. (2016). "La « déconstruction créatrice », une démarche de recherche en Supply Chain Management ", Rencontres Internationales de la Recherche en Logistique, Lausanne, Septembre.

Camman C., Guieu G., Livolsi L., Monnet M. (2013). "Les stratégies d'acteurs dans la mutualisation logistique ", Logistique \& Management, vol. 21, n`3, p. 57-75.

Camman C. et Livolsi L. (2009). « Le Category management, un vecteur de transversalité ? Le cas du Groupe Carrefour », Management \& Avenir, vol. 24, n4, p. 146-162.

Choi T.Y., Dooley K., Rungtusanatham M. (2001). "Supply networks and complex adaptive systems: control versus emergence", Journal of Operations Management, vol. 19, n³, p. 351366.

Corbett-Etchevers I. (2011). "De Candide à Zarathoustra », Revue Française de Gestion, $\mathrm{n}^{\circ} 216$, p. 129-144.

Cox A. (2001). "Understanding buyer and supplier power: A framework for procurement and supply competence", Journal of Supply Chain Management, vol. 37, n², p.8-15.

Cox A., Chicksand D. (2007). "The power regimes methodology: Differentiation strategies for achieving sustainable business success", Management Online Review, p.1-10.

Dyer J.H. et Singh H. (1998). "The relational view: Cooperative strategy and sources of interorganizational competitive advantage ", Academy of management review, vol. 23, $\mathrm{n}^{\circ} 4$, p.660-679.

Fernie S. et Tennant S. (2013). "The non-adoption of Supply Chain Management", Construction Management and Economics, vol. 31, n¹0, p. 1038 - 1058.

Fournier V. et Grey C. (2000). «At a Critical Moment: Conditions and Prospects for Critical Management Studies », Human Relations, vol. 1, n53, p. 7-32.

Giddens A. (1984). The constitution of society, Polity Press, Cambridge.

Gold S. et Schleper M.C. (2017). "A pathway towards true sustainability: A recognition foundation of sustainable supply chain management", European Management Journal, vol. $35, \mathrm{n}^{\circ} 4$, p. $425-429$.

Golsorkhi D., Huault I. et Leca B. (2009). Les études critiques en management. Une perspective française, Presses Universitaires de France, Paris.

Habermas J. (1979). Connaissance et intérêt, Gallimard, Paris. (1 ${ }^{\text {ère }}$ édition en 1976).

Harvey D. (1989). The Condition of Postmodernity, Basil Blackwell, Cambridge, MA.

Higgins M. et Ellis N. (2009). "Ethics and industrial networks: a Levinasian approach towards the study of justice in the supply chain", 25th Conference of the Industrial Marketing \& Purchasing Group, Marseilles, France.

Ketchen D.J. et Hult G.T. (2011). "Buiding Theory about Supply Chain Management: Some Tools from Organizational Sciences”, Journal of Supply Chain Management, vol. 47, n², p.12-18

Lecocq X. (2002). «Contribution à une réflexion sur l'articulation des niveaux d'analyse en sciences de gestion », Questions de méthodes en sciences de gestion, in Mourgues N., AllardPoési F., Abdelmajid A., Charreire-Petit S. et Le Goff J. (Dir), EMS, p.173-192.

Livolsi L. (2009). Le supply chain management: synthèse et propositions, Conférence de l'Association Internationale de Management Stratégique, Grenoble.

Matthews L., Power D., Touboulic A., Marques L. (2016). "Building Bridges: Towards Alternative Theory of Sustainable Supply Chain Management", Journal of Supply Chain Management, vol. 52, $\mathrm{n}^{\circ} 1$.

McCarthy L., Touboulic A., Matthews L. (2018). "Voiceless but empowered farmers in corporate supply chains: Contradictory imagery and instrumental approach to empowerment", Organization, vol. 25, n5, p. 609-635. 
Mcfarland R.G. et Payan J.M. (2008). « Supply Chain Contagion », Journal of Marketing, vol. 72, March, p. 63-79.

Meehan J., Touboulic A., Walker H. (2016). "Time to get real: The case for critical action research in purchasing and supply management", Journal of Purchasing and Supply Management, vol. 22, $\mathrm{n}^{\circ} 4$, p. 255-257.

New S. (2004). "Supply Chains: Construction and legitimation", in New S. and R. Westbrook Eds., Understanding Supply Chains. Concepts critiques and Futures, Oxford University Press, p. 69-108.

Nielson B. (2014). "Beyond Kulturkritik: Along the Supply Chain of Contemporary Capitalism", Culture Unbound. Journal of Current Cultural Research, vol. 6, n¹, p. 77-93.

Noireaux V. et Poirel C. (2014). "Contre-pouvoir et stratégies collectives. Comment contrer le pouvoir d'un puissant partenaire dans le canal de distribution ?", Revue Française de Gestion, $\mathrm{n}^{\circ} 240$, p. 81-95.

Oliveira N. et Lumineau F. (2019). "The Dark Side of Interorganizational Relationships: An Integrative Review and Research Agenda", Journal of Management, vol. 45, ${ }^{\circ} 1, \mathrm{n}^{\circ}, \mathrm{p} .231$ 161

Prieto-Carron M. (2008) "Women workers, industrialization, global supply chains and corporate codes of conduct", Journal of Business Ethics, vol. 83, $\mathrm{n}^{\circ} 1$, p.5.

Reimann F. et Ketchen D.J. (2017). "Power in supply chain management", Journal of Supply Chain Management, vol. 53, $\mathrm{n}^{\circ}$ 2, p.3-9.

Rotaru K., Leonid C, Flitman A. (2014). "Can critical realism enable a journey from description to understanding in operations and supply chain management?", Supply Chain Management: An International Journal, vol.19, $\mathrm{n}^{\circ} 2$, p. 117-125.

Schorsch T., Wallenburg C. M., Wieland A. (2017). "The human factor in SCM: introducing a meta-theory of behavioral supply chain management", International Journal of Physical Distribution \& Logistics Management, vol. 47, n², p. 238-262.

Spicer A., Alvesson M., Kärreman D. (2009). "Critical performativity: The unfinished business of critical management studies », Human relations, vol. 62, n 4, p. 537-560.

Touboulic A., Matthews L., Marques L. (2018). "On the road to carbon reduction in a food supply network: a complex adaptive systems perspective", Supply Chain Management: An International Journal, vol. 23, n4, p.313-335.

Touboulic A. et Walker H. (2015). "A relational, transformative and engaged approach to sustainable supply chain management: The potential of action research", Human Relations, vol. $69, n^{\circ} 2$, p. $301-343$.

Tsing A., (2009). "Supply Chains and the Human Condition", Rethinking Marxism: A journal of Economics, Culture \& Society, vol. 21, n², p. 148-176.

Villena V.H., Revilla E., Choi T.Y. (2011). "The dark side of buyer-supplier relationships: A social capital perspective”, Journal of Operations management, vol. 29, nº 6 , p. 561-576. 